DOE/ER/45431---2

DE92 040855

\title{
FIRST-PRINCIPLES STUDIES OF PHASE STABILITY \\ AND THE STRUCTURAL AND DYNAMICAL PROPERTIES \\ OF METAL HYDRIDES
}

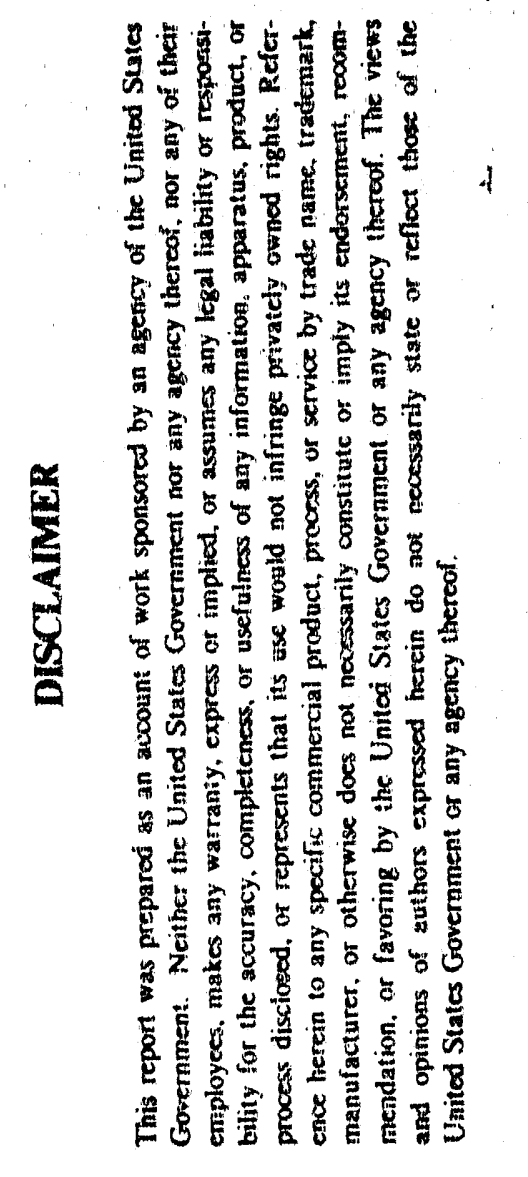

\author{
Progress Report
}

September 15, 1991 - September 14, 1992

M. Y. Chou

School of Physics

Georgia Institute of Technology

Atlanta, GA 30332-0430

April 1992 PREPARED FOR THE U.S. DEPARTMENT OF ENERGY
UNDER GRANT NUMBER DE-FG05-90ER45431

Recoived by OSTI

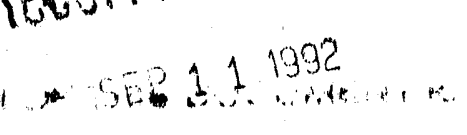




\section{TABLE OF CONTENTS}

Page

PROGRESS REPORT $(9 / 15 / 91-9 / 14 / 92) \ldots \ldots \ldots$

a. Calculation of the Structural Properties of Yttrium . . . . . . . . . . . 1

b. Dynamical and Pairing Properties of $\alpha-Y H_{x}$. . . . . . . . . . . . 2

c. Electronic and Structural Properties of $Y \mathrm{H}_{2}$ and $Y \mathrm{H}_{3}$. . . . . . . . . . 3

d. Phase Diagram of Hydrogen or Ru(000) . . . . . . . . . . . . . . . . . 3

SUMMARY OF FUTURE RESEARCH . . . . . . . . . . . . . 5

a. Peierls Distortion in Hexagonal $Y H_{3}$. . . . . . . . . . . . . 5

b. Study of Hydrogen in Niobium and Palladium . . . . . . . . . . . . . . . 6

c. List of Personnel . . . . . . . . . . . . . . . . . . . . . . . . . . . . 7

d. List of Other Federal Support . . . . . . . . . . . . . . . . . . . . . . 8

e. Budget $(9 / 15 / 92-9 / 14 / 93) \ldots \ldots . \ldots . . \ldots 9$

f. Statement of Cost Sharing . . . . . . . . . . . . . . . . . 10

APPENDICES

A. Abstracts Submitted for the 1992 APS March Meeting

B. Reprint "Pseudopotential Plane-Wave Calculation of the Structural Properties of Yttrium" 
PROGRESS REPORT $(9 / 15 / 91-9 / 14 / 92)$

We have been concentrating on the study of the variou phases of the yttrium-hydrogen system. Elemental yttrium is in the hexagonal close-packed (hcp) structure. For a low concentration of hydrogen, a solid solution is formed (the so-called $\alpha$ phase) with hydrogen occupying the tetrahedral sites in the metal lattice. When more hydrogen is put in, a dihydride phase starts to form and the metal lattice transforms to the face-centered cubic $(\mathrm{fcc})$ structure. For even a higher hydrogen concentration, a hexagonal lattice is again favored and in the trihydride pinase the yttrium atoms are back to the hep structure, but the positions of hydrogen atoms exhibit some unusual relaxations. One major finding of our study is that the structural and dynamic properties of this system are strongly coupled to the electronic structure and the simplified so-called "proton" and "anion" models will not be able to account for the rich phenomena observed experimentally. The electronic structure is in fact the driving force for the existence of various different phases.

We also performed a thermodynamic study of hydrogen chemisorption on the Ru(0001) surface. The phase diagram was calculated and a concentration-dependent effective pair interaction is found to be needed in order to explain the asymmetry in the phase diagram. Our results are summarized below.

\section{a. Calculation of the Structural Properties of Yttrium}

With the recent development of soft pseudopotentials, we chose to use the plane wave basis set to carry out the calculation. The advantages of using this complete and orthogonal basis set have been well recognized. Previous problems have been associated with the slow convergence for transition metals. Therefore we first tested the computational methods by studying the structural properties of elemental yttrium and the results were encouraging. Good convergence is obtained with an energy cutoff of 30-40 Ry. It is also found that 
special care is necessary to handle the outer core in these early transition metals. The effect of using different exchange-correlation potentials is also investigated. The final paper entitled "Pseudopotential Plane-Wave Calculation of the Structural Properties of Yttrium" has been published in the Physical Review B, vol. 44, F. 10339 (1991).

\section{b. Dynamical and Pairing Properties of $\alpha-Y H_{x}$}

It is well established that hydrogen dissolved in the hcp rare-earth metals exhibits unusual short-ranged ordering which is closely related to the high stability of the $\alpha$ phase at low temperature. In addition, there have been quite a few neutron scattering results available for the local vibrational modes in this system. We consider first the ordered structure $Y H_{0.5}$ with one hydrogen per unit cell occupying two different kinds of interstitial sites: the tetrahedral $(\mathrm{T})$ and octahedral $(\mathrm{O})$ sites. A lattice expansion of $6 \%$ is found in the system due to the presence of hydrogen and results from the minimization of the kinetic energy. The binding energies are calculated to be $4.27 \mathrm{eV}$ and $4.10 \mathrm{eV}$ for the tetrahedral and octahedral sites respectively, with a difference of about $170 \mathrm{meV}$. Our result also reveals that the tetrahedral site has a lower energy than the octahedral site. Hence the occupation of tetrahedral sites is energetically more favorable. This is consistent with the conclusion of neutron scattering experiments.

We have also studied the dynamical properties of this system and calculated the local vibrational frequency from the potential surface for the protons. The anharnonic and arisotropic nature of the potential surface is well characterized in the study. Interesting results are found when we move the hydrogen atom along the c-axis between two adjacent tetrahedral sites. The potential energy curve consists of two anharmonic wells with a barrier of about $190 \mathrm{meV}$. This double-well potential forms a 1-D quantum system and we can calculate the eigenvalues by solving the Schrodinger equation for the proton. From these eigenvalues, the local vibrational modes can be identified. We found the fundamen. 
tal frequency to be around $100 \mathrm{meV}$ which is in good agreement with the experimental observation.

In addition, the pairing energy is calculated for the first time from tirst principles and it shows that pairing of hydrogen through the intermediate metal atom is indeed energetically favorable. The calclulated energy difference (50 meV) is much smaller than. the results from previous less-accurate estimates. We are currently analyzing the details of the mechanism and hope to provide a unified picture for hydrogen in rare-earth metals.

The final paper is being written and is expected to be finished in the summer of 1992 .

\section{c. Electronic and Structural Properties of $\mathrm{YH}_{2}$ and $\mathrm{YH}_{3}$}

In this study, we are mainly interested in the bonding characteristics of hydrogen in these high concentration phases. Depending upon the symmetry of the interstitial sites, the hydrogen $s$ electron will interact with different states of the metal $d$ orbitals. By examining the decomposed density of states, we confirmed the chemical picture that the tetrahedral (octahedral) hydrogen interacts with the $t_{2 g}\left(e_{g}\right) d$ orbital. It was also found that the volume expansion from $Y$ to $Y H_{2}$ is mainly due to the minimization of the kinetic energy of the extra electrons, while the contraction from $\mathrm{YH}_{2}$ to $\mathrm{YH}_{3}$ comes from maximizing the s-d interaction at the octahedral site.

Another interesting quantity is the charge transfer in these system. It was found that a large charge transfer from yttrium to hydrogen occurs when $\mathrm{YH}_{2}$ is formed. Additional charge transfer in $\mathrm{YH}_{3}$ compared with $\mathrm{YH}_{2}$ is much less significant. The results of this part of study is also being written up.

\section{d. Phase Diagram of Hydrogen on Ru(0001)}

It is commonly believed that chemisorbed hydrogen occupies the threefold hollow sites on close-packed metal surfaces. As temperature and concentration vary, different ordered 
and disordered phases may become stable. Assumirig a two-dimensional lattice gas model, we studied the thermodynamical properties of this chemisorption system. The cluster variation method (CVM) was used to evaluate the free energy of the system. By minimizing the free energy at a given concentration, the temperature versus concentration phase diagram is obtained. At high temperature, hydrogen atoms occupy the hollow sites randomly and form a disordered phase. As temperature is lowered, several ordered structures are found, including the $2 \times 2, \sqrt{3} \times \sqrt{3}$, and $2 \times 1$ structures. The maximal transition tempuratures coincide with the ideal concentration values of $1 / 4,1 / 3$, and $1 / 2$ respectively.

In order to reproduce the experimental asymmetric phase diagram, it is found that a repulsive second nearest neighbor interaction is needed and it is also concentration dependent. By assuming a simple piecewise lirear dependence on the chemical potential, we reconstructed an asymmetric phase diagram which agrees well with experiment. The model studied can be applied to the H/Pd(111) system directly and can be easily generalized for other close-packed metal surfaces.

A manuscript is being prepared to summarize these findings for the phase diagram. 


\section{SUMMARY OF FUTURE RESEARCH}

\section{a. Peierls Distortion in Hexagonal $Y \mathrm{H}_{3}$}

It has been found from our calculation that in $\mathrm{YH}_{3}$ the octahedral hydrogen atoms move down to the metal plane in order to offset the relaxations of tetrahedral hydrogen. If the periodicity of the compound is the same as the hcp metal, the material is a semimetal, with electron pockets located at the $\mathrm{K}$ points and holes at $\Gamma$. The Fermi surface nesting feature will be responsible for certain soft phonon modes in the high temperature phase. In addition, the system is subject to a Peierls instability and a gap can be opened up when the symmetry is broken. The ground state at low temperature is expected to be a semiconductor.

We plan to search for the optimal relaxation of hydrogen atoms as well as to examine the electronic structure in details. From some earlier neutron diffraction data on $\mathrm{HoD}_{3}$, it is expected that the unit cell for $Y H_{3}$ is to be tripled to a $\sqrt{3} \times \sqrt{3}$ lattice. The energy of the "frozen in" local vibrational wave of hydrogen is to be minimized to determine the nature and amplitude of the displacements. This involves a series of total energy calculations as well as investigations of the electronic structure. Sorne preliminary results have shown that a gap indeed exists. This will be an unusual case of a two-dimensional Peierls distortion.

After we obtain the static ground state information, we plan to develop a model for the semiconductor-metal transition at finite temperature. This needs to take into account the electronic free energy and the elastic energy within the same framework. We hope to show that the transition ternperature is high, so the semicondutor phase is observed at room temperature as for $\mathrm{HoD}_{3}$. This will be a new finding in metal hydrides that the hydrogen displacements from symmetric interstitial sites can be quite substantial and the interplay of the electronic and structural properties is of fundamental importance in these materials. 


\section{b. Study of Hydrogen in Niobium and Palladium}

After the study of hydrogen in hcp yttrium, we will further investigate the hydrogen interaction with metals in the body-centered and face-centered cubic (bcc and fcc) structures. The prototype system will be niobium and palladium respectively. Both metals have stable binary hydrides and have extensive experimental data available. Since the three dimensional lattice will be changed, the optimal interstitial site for hydrogen will also be different. This will in turn affect the diffusion behavior, vibrational modes, possibility of ordering, and much more. The purpose of the study will be to identify the trend in various physical properties when the number of $d$ electrons is increased and crystal structure changed.

The binding properties will be the first one we like to investigate using the pseudopotential method within the local-density functional formalism. Usually the octahedral site is favored in fcc metals while the tetrahedral site is favored in bcc metals. It may be explained by a solid sphere packing model with a fixed effective radius for the hydrogen atom. We wish to examine this question and study the physical basis of the success of this packing model from the information obtained in our first-principles studies for three different metals. In addition, a change of the hydrogen occupancy from the tetrahedral to the octahedral site occurs in the $N b H_{x}$ system when the metal is compressed. This phase transition under high pressure is closely related to the incompressibility of hydrogen in metals and is worth further studies. We can also look into the effect of uniaxial stress by decreasing the lattice constant in a chosen direction. In either case, the change in the electronic structure will be examined in details.

There are other interesting experimental results which require further theoretical investigations. For bcc metals such as niobium, the optical modes appear to be dispersionless and depend only weakly on the hydrogen concentration. These indicate that the restoring forces come mainly from the hydrogen-metal interaction and the hydrogen-hydrogen inter- 
action is relatively weak. On the contrary, the optical modes observed in the H-Pd system are lower in energy and exhibit a very significant wave-vector dispersion. In our total energy studies, we can move the atoms freely and evaluate the energies and HellmannFeynman forces for different atomic arrangements. This information can then be used to compute the phonon energy at high symmetry points by the frozen-phonon approximation or by solving for the atomic motion in a potential well. The results will shed some light on the physical origin of the difference in the interaction strength in different hydrides.

Hydrogen diffusion can be found over a large temperature range and for different isotopes $w$ ith the largest possible mass ratios. These provide a good experimental data base for the development and testing of the quanturn theory of diffusion in statistical mechanics. A knowledge of the interactions between the interstitial hydrogen atoms and the metal host and among the hydrogen atoms themselves are essential in order to discuss the diffusion process. In particular, the room-temperature diffusion coefficient for hydrogen in niobium is $5-10$ orders of magnitude larger than that for other interstitials like oxygen and nitrogen. This is expected to be related to small diffusion barriers which can only be reliably examined in a total energy study. We plan to map out the classical energy surface for the three metals in different crystal structures and investigate the difference. We can also easily examine the influence of hydrostatic pressure on this energy surface by changing the lattice scale. This information, together with the vibrational spectra, will be the input for further statistical-mechanical model studies for the diffusion mechanism.

\section{c. List of Personnel}

Principal Investigator: M. Y. Chou

Graduate Student: Yan Wang (September 1990 - present)

Postdoctoral Fellow: S. N. Sun (August 1991 - present) 


\author{
Abstract Submitted \\ for the March 1992 Meeting of the \\ American Physical Society
}

March 16-20,1992

Suggested Session Title:

Sorting Category $30 \mathrm{e}$

Chemisorption and phase transitions

Ordered Structures and Phase Transitions of Hydrogen on Close-packed Transition Metal Surfaces.* SHENG N. SUN and M.Y. CHOU, School of Physics, Georgia Institute of Technology, Atlanta, GA 30332-0430. - We study possible ordered structures of hydrogen atoms on close-. packed transition metal surfaces. Assuming a lattice gas model for the chemisorbed hydrogen atoms, the ground state of the system is then uniquely determined by the interactions among hydrogen atoms and lattice symmetries. Ground state analysis including the nearest and the next-to-nearest neighbor interactions shows several ordered structures at different coverages. Among the obtained structures, $(\sqrt{3} \times \sqrt{3}) R 30^{\circ}$ at a coverage of $\theta=0.33,(2 \times 1)$ at $\theta=0.50$, and $(2 \times 2)$ at $\theta=0.75$ are observed experimently for $\mathrm{H}$ on $\mathrm{Ru}(001)$ by Sokolowski et al. recently. ${ }^{1}$ The phase transitions to disorded phases at higher temperatures are being studied by the cluster variational method for the lattice gas system.

* Supported by the DOE grand No. DE-FG05-90ER45431.

${ }^{1}$ M. Sokolowski, T. Koch and H. Pfnür, Surf. Sci. 243, 261 (1991).

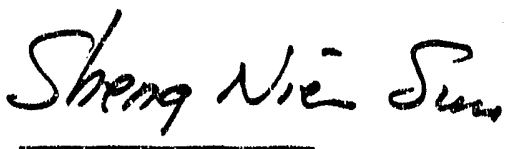

Sheng Nien Sun

School of Physics

Georgia Tech

Atlanta, GA 30332-0430

APS Member MSU57577 


\section{Abstract Submitted for the 1992 March Meeting of the American Physical Society \\ 16-20 March, 1992}

HYDROGEN IN METALS

Sorting Category: $15(d)$

Theoretical Study of the High Concentration

Phases of Hydrogen in Yttrium.* YAN WANG and

M. Y. CHOU, Georgia Tech. --- The calculations based on the local density theory and the pseudopotential planewave method showed that, from energetics consideration, the yttrium metal will transfer from hcp to fcc structure in order to accommodate more hydrogen. The bonding characters and the total energies associated with different interstitial sites and various hydrogen compositions are investigated. It is found that partial charge transfer occurs which is studied from the self-consistent results.By modeling the system with a three-dimensional lattice gas,implications of phase transitions will be discussed.

* Supported by DOE and Pittsburgh Supercomputing Center.

Prefer Standard Session

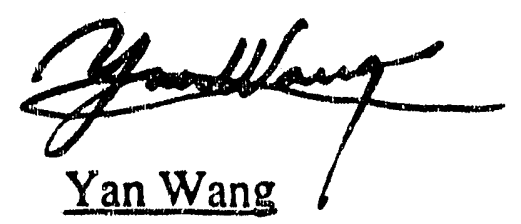

School of Physics

Georgia Institute of Technology Atlanta. GA 30332-0430 


$$
\begin{gathered}
\text { Abstract Submitted } \\
\text { for the March 1992 Meeting of the } \\
\text { American Physical Socie } y \\
\text { March 16-20, } 1992 \\
\hline
\end{gathered}
$$

Suggested Session Title:

Phonons and Dynamical Properties

Sorting Category 21b.

Calculation of the Phonon Spectra from First Principles.* Siqing WEI, M. Y. CHOU, School of Physics, Georgia Institute of Technology, Atlanta, GA 30332-0430. We developed a scheme to deduce the individual atomic force constant from the known data obtained from the LDA supercell calculations. The idea is to maximize the information acquired from a supercell calculation and overcome the previous difficulties. Our scheme is based on the idea by Kunc et al. ${ }^{1}$ where the inter-plane force constants were calculated. We further analysis their inter-plane force constants and derive the individual inter-atomic force constants. With this approach, simple supercell calculations will provide all the information needed to construct the entire dynamical matrix for an arbitary wave vector, hence the full phonon spectra over the entire Brillioun zone can be calculated. An calculation for Germanium is shown as an example of this approach.

* Supported partly by DOE and NSF.

${ }^{1}$ Kunc and Dacosta, Phys. Rev. B32, 2010 (1985).

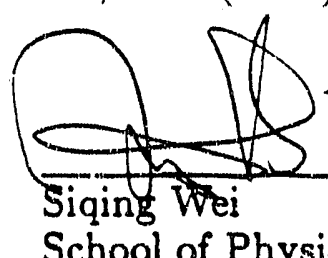

School of Physics

Georgia Tech

Atlanta, GA 30332-0430

APS Member MWE269447

Prefer Standard Session

P. G. Dacosta

Suggested Chairperson 

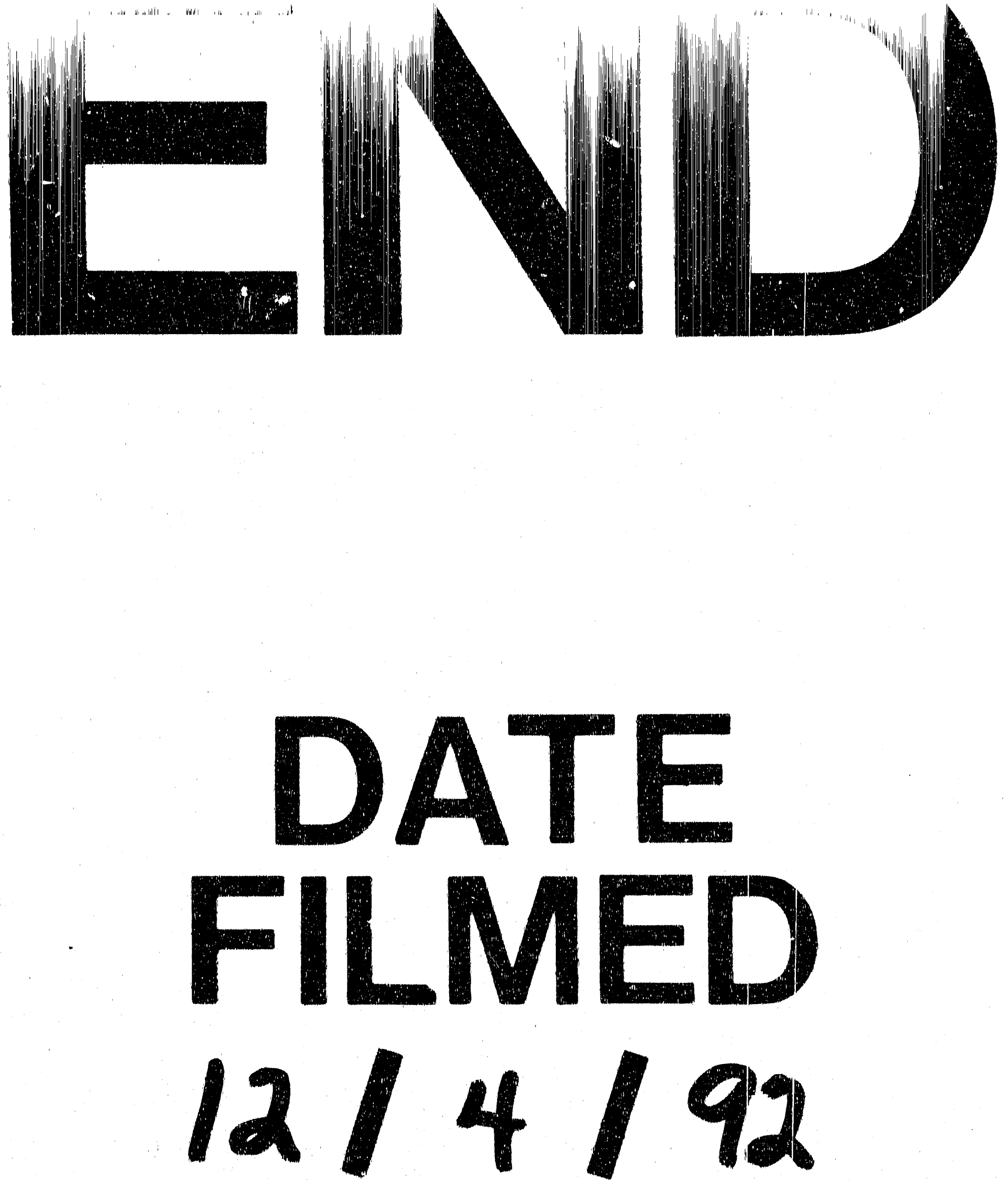

$\$$ 
